\title{
REVISIÓN CRÍTICA DE LOS CRITERIOS DE IDENTIFICACIÓN DE LOS COMPLEMENTOS DE UNA FORMA NOMINAL
}

\author{
Fco. Javier Tamayo Morillo
}

\begin{abstract}
The specification of the complementation possibilities of a lexical item is nowadays felt to be a major part of the description of its syntactic behaviour. This information is relevant in two respects mainly: first, because lexical units are categorized into subclasses on the basis of the number and nature of their complements; and second, because it ensures the discreteness of that lexical item versus others that may share other aspects of its behaviour. However, though any lexical item can be characterized with respect to the range of complements that it takes, the discussion of noun complementation has received less attention than the complementation of verbs and adjectives in linguistic theory. One of the reasons for this is that grammarians have had difficulty in formulating tests for identifying noun complements. It is the purpose of this paper to review a number of the criteria that have been proposed for distinguishing between complements and adjuncts, mostly with regard to verb dependents, and see if they are applicable in the case of noun dependents.
\end{abstract}

Dentro de los estudios sintácticos actuales, una de las líneas de investigación que está resultando más productiva es la que tiene como objeto el examen de las relaciones sintagmáticas que contraen los elementos que coaparecen en las expresiones lingüísticas. Los trabajos que se vienen realizando en esta dirección exploran sobre todo el vínculo existente entre el núcleo de estas expresiones y uno de los tipos de elementos que pueden estar subordinados a éste: sus complementos. La información que se desprende de este apartado de la descripción gramatical es útil en muchos sentidos. Así, por ejemplo, permite la identificación del modelo de complementación propio de ese núcleo, una propiedad sintáctica del mismo que se considera esencial por dos motivos fundamentalmente. En primer lugar, porque hace posible una caracterización de esta unidad que facilita su inclusión en alguna de las subcategorías que se distinguen dentro de la categoría a la que pertenece ${ }^{1}$. En segundo lugar, porque se trata de un dato que sirve para componer una definición lexicográfica más exacta de dicha unidad ${ }^{2}$.

\footnotetext{
${ }^{1}$ Garrudo Carabias $(1991,1996)$, por ejemplo, establece una tipología verbal completiva sobre la base de los siguientes modelos mayores: intransitivo, transitivo, adjuntado y copulativo.

${ }^{2}$ A este respecto, cabe señalar cómo muy a menudo se ha podido comprobar que los datos que se expresan en los 
Pero aun reconociendo la importancia que, como acabamos de manifestar, se le ha otorgado al concepto de complemento, hemos de señalar que los gramáticos suelen discrepar a la hora de reflejar la pertinencia que el mismo tiene en el esclarecimiento de los rasgos sintácticos de los elementos pertenecientes a las distintas categorías léxicas. De esta manera, hay que señalar que existe un acuerdo bastante generalizado en cuanto a la trascendencia que esta noción tiene en el terreno de la caracterización de las formas verbales y adjetivales. Llama la atención, sin embargo, la falta de conformidad en lo concerniente al valor que tiene en el ámbito de los nombres. En este apartado queremos destacar la opinión de una serie de lingüistas (véanse, por ejemplo, Colen (1984:69) y Mackenzie (1985)) que se manifiestan totalmente contrarios a catalogar los adyacentes nominales con arreglo al mencionado instrumento conceptual.

Ante este estado de cosas, nuestro objetivo en este trabajo es mostrar que esta etiqueta funcional puede trasladarse al ámbito de los nombres. Con tal fin vamos a repasar las pruebas y criterios que con más frecuencia han sido empleados en las esferas verbal y adjetival para identificar las unidades lingüísticas que desempeñan este papel, comprobando su idoneidad en este otro terreno. Los mecanismos que revisaremos, de carácter formal en su totalidad, son los siguientes: la prueba de la supresión, la pronominalización, el orden de palabras, la coordinación y la prueba de la libre adición de dependientes. Veamos, pues, hasta qué punto cada uno de estos procedimientos puede ayudarnos en nuestra tarea de distinguir los complementos de una forma nominal.

La prueba de la supresión consiste en estudiar los efectos que tiene la eliminación en una secuencia gramatical de la unidad lingüística cuyo rango sintáctico estamos verificando. De este modo, se hace patente uno de los rasgos que con más asiduidad se les ha atribuido a los complementos de una pieza léxica: su carácter obligatorio. Entre los lingüistas que ponen de relieve la validez de este requisito se halla Lyons (1968:345):

(1) The difference between an adjunct and a complement is, in principle, quite clear: the former is an optional (extranuclear) constituent, and the latter an obligatory (nuclear) constituent of the sentence.

De acuerdo con este punto de vista, los complementos se presentan como aquellos componentes de una estructura sintáctica cuya aparición en la misma es exigida por el elemento del que dependen. Es preciso que aclaremos que la obligatoriedad de estas unidades puede ser enfocada desde una perspectiva tanto formal como nocional. Así, por un lado, un planteamiento formal o sintáctico de este criterio sirve para identificar como obligatorios aquellos constituyentes de una construcción sintáctica cuya eliminación altera

diccionarios de uso de una lengua, de índole semántica básicamente, no dejan mostrar con total precisión los distintos lexemas que se representan mediante una misma forma. Esto ha hecho que un gran número de autores, entre ellos Paul (1982), se pronuncien a favor de la incorporación de esta información en los trabajos lexicográficos. La obra de Garrudo Carabias $(1991,1996)$, referencia importante para los estudios que se inicien en esta línea, permite valorar el verdadero alcance de estas propuestas. 
su gramaticalidad; un acercamiento semántico, por otro lado, califica como obligatorias las unidades que aportan información que afecta al significado del núcleo de dicha construcción.

La adopción de este criterio, sin embargo, plantea en numerosas ocasiones problemas. Estos problemas se concretan en la existencia de complementos que no se adecúan a la definición propuesta al ser dispensables. Esta situación, que es perceptible en el ámbito de cualquier categoría léxica mayor, se deja sentir de una forma más palpable en la esfera de los nombres. En efecto, como suele señalar un gran número de autores, los complementos de un nombre son, por esencia, sintácticamente opcionales ${ }^{3}$. Para conocer los factores determinantes de esta situación hemos de dirigir nuestra atención básicamente hacia dos aspectos: el valor específico de los nombres y el papel semántico-pragmático de la secuencia nominal en la oración. Detengámonos ahora en la consideración del primero de estos aspectos.

Un rasgo esencial de la caracterización sintáctica de los nombres es el hecho de que pueden funcionar como elementos autónomos. Antes de ilustrar esta circunstancia con la aportación de algún especialista, nos interesa explicar el porqué de este comportamiento que es privativo de los miembros de esta categoría sintáctica.

Esta conducta encuentra su justificación en el tipo de significado que estas unidades expresan. Veamos la definición semántica que de los nombres, adjetivos y verbos se han propuesto en las teorías gramaticales tradicionales. Ésta ha sido resumida por Lyons (1977:439) en las siguientes líneas:

(2) Nouns are traditionally said to denote entities, verbs and adjectives to denote what we may refer to here as first-order properties (in a very general sense of the term 'property')...

Se fija, según podemos observar, una clara distinción entre las realidades extralingüísticas denotadas por estos elementos. Los nombres, por un lado, señalan objetos que se conciben como independientes. Frente a éstos, los verbos y los adjetivos expresan conceptos dependientes.

Pues bien, tales propiedades tienen su reflejo en el plano sintáctico donde, como hemos indicado, los nombres manifiestan igualmente un comportamiento muy particular. En este sentido, convendría destacar las ideas aportadas por Jespersen (1909-49, II:2-8) al respecto. Este autor, al afrontar la cuestión de la clasificación de las palabras, declaró que ésta se podía efectuar desde dos puntos de vista. Según él, existía una primera posibilidad de análisis, sobre la que no nos detendremos, en la que las mismas se consideraban de acuerdo

\footnotetext{
${ }^{3}$ Este tipo de planteamiento es el que propugnan, entre otros, Jackendoff (1977:58), Anderson (1979:134), Leech (1981:184-6), Huddleston (1984:263), Radford (1988:180), y Herbst (1988).
} 
con sus rasgos formales. Ésta se habría de completar con el estudio de su comportamiento dentro de la construcción sintáctica en la que aparecían. Así, él (1909-49, II:2) distinguía entre lo que eran términos primarios, términos secundarios y términos terciarios:

(3) We are thus led to establish different ranks of words according to their mutual relations as defined or defining. In the combination extremely hot weather, weather may be called a primary word or a principal; hot, which defines weather, is a secondary word or an adjunct; and extremely, which defines hot is a tertiary word or a subjunct.

Desde esta perspectiva funcional, pues, los nombres se caracterizan por actuar habitualmente como términos primarios, mientras que los adjetivos y los verbos lo hacen como términos secundarios. Quiere esto decir que el empleo en una oración de una lexía perteneciente a una de estas dos últimas clases de palabras presupone siempre el uso de algún nombre. Cualquier adjetivo, por una parte, necesita un nombre que identifique la entidad que es soporte del rasgo que dicho adjetivo manifiesta. Como se puede apreciar en estos ejemplos la posición del adjetivo respecto a dicho nombre es muy variada:

(4) (a) The police have abundant proof of his guilt.

(b) I'm looking for a piece of land abundant in minerals.

(c) The supply of food they sent us is abundant.

Igualmente, los verbos precisan de elementos que presenten los entes participantes en los estados de cosas que designan:

(5) That girl wrote my friend a long letter.

Por lo que se refiere al segundo de los aspectos anteriormente citados, hemos de significar que los componentes de la oración, considerados desde un punto de vista semántico-pragmático, son normalmente agrupados en dos clases de unidades: las expresiones referenciales y las expresiones predicativas. Con el primero de estos vocablos se designan aquellas unidades lingüísticas que aluden a las entidades que son el centro de un comentario. El segundo señala la función de las que designan propiedades de las entidades o relaciones entre ellas. Pues bien, los sintagmas nominales suelen identificarse prototípicamente con la función referencial, lo cual explica también la opcionalidad de los complementos de los nombres. Efectivamente, al hacer uso de un grupo nominal intentamos remitir a una determinada entidad del mundo. En este sentido, el papel que desempeñan los modificadores en esta estructura es el de proporcionar los datos precisos que, junto con la información aportada por el nombre, nos ayudan a tal fin. Así pues, es evidente que su presencia en el grupo nominal está condicionada por factores de índole comunicativa. El hablante recurre a ellos en la medida en que contribuyen a facilitar la identificación del referente concreto de la expresión nominal. Estamos, consiguientemente, ante otro hecho que repercute en la conducta sintáctica de los modificadores del nombre, 
estatus que, no olvidemos, comparten sus complementos y adjuntos. Ésta es, por ejemplo, la opinión de Quirk et alii (1985:65), quienes consideran que:

(6) Modification... is a largely optional function.... Semantically, modifiers add 'descriptive' information to the head, often restricting the reference of the head. Thus a green table has a more specific meaning than a table, and very tall has a more specific meaning than tall.... In this respect, modifiers in phrases parallel adverbials in the clause.

Además de la propiedad a la que nos acabamos de referir, la prueba de la supresión pone al descubierto otra de las características que se les atribuye a los complementos: la latencia. Este requisito indica lo siguiente: si un complemento no se halla recogido expresamente en una secuencia lingüística, debe estarlo al menos de una manera tácita. A diferencia del anterior, este rasgo sí resulta sumamente útil para determinar si un dependiente nominal tiene o no rango de complemento. Veamos cómo.

Puesto que existen dos modos de aproximarse a este asunto, uno sintáctico y otro semántico, vamos a empezar por examinar la posibilidad de definir los complementos de un nombre desde la vertiente sintáctica. Así, desde este punto de vista se dice que son complementos todas aquellas unidades lingüísticas eliminadas de una secuencia gramatical que las propiedades estructurales de dicha construcción obliga a recuperar. Obsérvese que, definida en estos términos, la noción de latencia nos situaría en el ámbito de la elipsis, fenómeno que Halliday y Hasan (1976:143) describen con estas palabras:

(7) When we talk of ellipsis, we are not referring to any and every instance in which there is some information that the speaker has to supply from his own evidence. That would apply to practically every sentence that is ever spoken or written, and would be of no help in explaining the nature of a text. We are referring specifically to sentences, clauses, etc whose structure is such as to presuppose some preceding item, which then serves as the source of the missing information.

Estudiemos ahora la aplicación que esto puede tener en el tema que nos ocupa. Habida cuenta de que, como hemos subrayado con anterioridad, los complementos de un nombre son constituyentes opcionales del sintagma nominal, esto es, su ausencia no afecta para nada a la estructura básica de este tipo de construcciones, resulta evidente que un acercamiento que se sustente en los presupuestos que se acaban de exponer debe ser rechazado ${ }^{4}$.

\footnotetext{
${ }^{4}$ Quirk et alii (1985:902) confirman esta idea cuando afirman: "Since postmodifiers are in general optional, it is difficult to recognize ellipsis in this position". Halliday y Hasan (1976:147-66), que tratan extensamente el tema de la elipsis en el sintagma nominal, son también partidarios de esta opinión. Así, siguiendo este criterio que señala que hay elipsis siempre que se omite un elemento estructuralmente necesario, ellos (1976:148) sostienen que: "Nominal ellipsis... involves the upgrading of a word functioning as Deictic, Numerative, Epithet or Classifier from the status of Modifier to the status of Head".
} 
Vistas, por tanto, las dificultades que entraña la definición de los complementos nominales con arreglo a una concepción formal de la noción de latencia, enfocaremos en este momento este tema desde una perspectiva semántica. Antes, no obstante, de abordar esta cuestión, hemos de establecer cuáles son las propiedades nocionales de los complementos. En este sentido, hemos de señalar que los gramáticos coinciden en hacer notar que estos elementos del plano sintáctico representan las unidades que se hallan contenidas en la caracterización significativa del núcleo léxico al que acompañan. Como ilustración de esto que decimos, recogemos la siguiente cita de Quirk et alii (1985:65):

(8) We reserve the term COMPLEMENTATION (as distinct from complement) for the function of a part of a phrase or clause which follows a word, and completes the specification of a meaning relationship which that word implies.

Examinemos, pues, la cuestión de la latencia de los complementos a la luz de estas consideraciones. Así, se dice que la elisión de una estructura completiva está sujeta a un requisito muy concreto: ésta siempre ha de sobreentenderse para que tenga sentido la construcción sintáctica en la que se ha suprimido. Refiriéndose a este hecho, Quirk et alii (1985:66) manifiestan que:

(9) Although complementing elements may be optional, such elements differ semantically from other optional elements... in that the omission of complementation... implies that some element of meaning in a preceding word is 'unsatisfied', and therefore has to be provided through context.

Fijémonos en los sintagmas nominales que hemos colocado entre corchetes en las siguientes oraciones (los núcleos aparecen en negrita; los complementos, en cursiva; y los adjuntos en tipo normal):

(10) (a) They have elected [SNthe candidate for this job from Scotland].

(b) I don't want to meet [SNthe friend of the woman responsible for that misunderstanding with long hair].

En ambos casos los complementos únicamente pueden ser suprimidos contextualmente:

(11) (a) They have elected [SNthe candidate from Scotland].

(b) I don't want to meet [sN the friend with long hair].

De acuerdo con lo que venimos sosteniendo, se puede decir que los nombres de estos ejemplos requieren la presencia, implícita o explícita, de elementos que especifican su alusión léxica. Es importante saber valorar la relevancia que estos complementos nominales tienen en estas expresiones. Así, si bien su ausencia, como ya hemos explicado, no nos puede lievar a emilir un juicio de agranaticalidad en ningunana de las oraciones, sí puedé hacer que las cataloguemos como inaceptables. Esto significa que una persona logrará 
comprender el sentido que tienen tales expresiones sólo si tiene acceso a la información a la que aludían estos elementos. Nada de esto ocurre, sin embargo, en:

(12) (a) They have elected [sN the candidate for this job].

(b) I don't want to meet [sN the friend of the woman responsible for that misunderstanding].

estructuras cuyo significado se conserva íntegro a pesar de la eliminación de los adjuntos $^{5}$.

Por consiguiente, los hechos analizados nos han permitido descubrir uno de los factores en los que puede sustentarse la identificación de los complementos nominales.

En las páginas anteriores hemos repasado el modo en el que el criterio de supresión puede servirnos para sacar a la luz diferencias entre los complementos y los adjuntos de una forma nominal. Continuando con la revisión de las pruebas que se han empleado para establecer esta distinción, nuestra exposición se detendrá ahora a explorar la posibilidad de una diferencia de carácter estructural entre estos elementos. Así, avanzamos que la evidencia de los datos que vamos a considerar pondrá de manifiesto que los complementos generalmente se ubican en un lugar más próximo al núcleo léxico del que dependen que aquel en el que se sitúan los adjuntos.

Para estudiar la cuestión que tenemos planteada vamos a recurrir a las ideas de la Teoría de la X-barra, dado que el formato que aquí se postula para el análisis de la estructura interna de una unidad sintagmática es eficaz para alcanzar nuestro objetivo ${ }^{6}$. He aquí, en su

${ }^{5}$ Hemos de advertir, no obstante, que los complementos de una forma nominal puedan estar sujetos a otro tipo de elisión: la elisión indefinida. Veamos el siguiente ejemplo:

(i) Everybody wanted to see the fountain pen of the student of chemistry.

Así, si bien cabe la posibilidad de que la eliminación de of chemistry dé lugar a una expresión en la que este elemento se halle latente, lo relevante en este caso es que, en contraste con las construcciones que acabamos de examinar, la eliminación de esta estructura completiva puede ser también de índole indefinida. Esto es lo que explica que en:

(ii) Everybody wanted to see the fountain pen of the student.

no haya necesidad de recuperar un complemento implícito.

Pero esto en absoluto quita validez a nuestra argumentación ya que, comoquiera que el complemento en este último ejemplo no se halla referido ni siquiera de manera tácita, consideramos que es más acertado sostener que el nombre student en (ii) no toma tal complemento. Este hecho sitúa este proceso de eliminación en el terreno de los mecanismos de cambio e innovación léxicos, en concreto en el dominio del conocido como conversión sintáctica (véanse Leech (1981:215-7) y Quirk et alii (1985:722-3 y 1558 y ss)). La supresión del complemento en el ejemplo analizado ha provocado un cambio en el tipo de complementación del nombre afectado por el mismo y, como consecuencia, una distinción de formas nominales homónimas.

${ }^{6}$ Véase Chomsky (1972), (1981), (1986a) y (1986b) para una aproximación a los principios de este módulo de la gramática generativa . Igualmente, en Jackendoff (1977), Sells (1985:27-33), Garnica Silva (1987), Horrocks (1987:56-68), Radford (1988:167-279) y Cook (1988:94-102) podemos encontrar una descripción más rigurosa de la misma. 
versión más estándar, la estructura que en esta teoría se atribuía a cualquier secuencia gramatical de índole sintagmática ${ }^{7}$ :

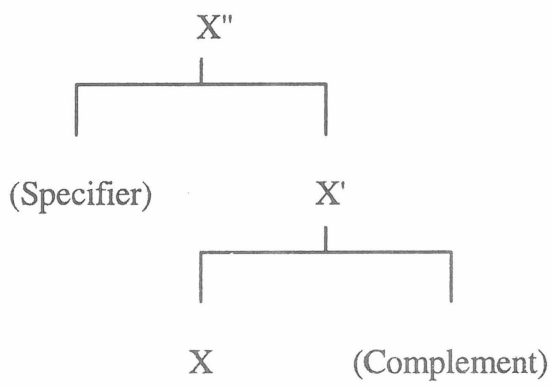

donde $X^{\prime \prime}, X^{\prime}$ y $X$ representan los distintos niveles que se distinguen en el análisis de dicha categoría.

Como puede comprobarse, la Teoría de la X-barra destaca que las categorías sintagmáticas están organizadas jerárquicamente. Así, se considera que éstas están integradas no por una secuencia lineal de elementos sino por distintos estratos de constituyentes. Concretamente, en el formato que hemos formulado unas líneas más arriba se reconocen tres niveles de proyección, los ya mencionados $X^{\prime \prime}, X^{\prime}$ y $X$. Los términos adyacentes del núcleo de estas categorías sintagmáticas aparecen vinculados a un nivel determinado según el papel que desempeñen en estas estructuras, esto es, especificador, adjunto o complemento. El objetivo fundamental de este apartado a partir de este instante será comprobar la solidez que tiene la defensa de este planteamiento en lo que respecta a la distinción entre los complementos y los adjuntos de una forma nominal. Para ello vamos a hacer uso de una serie de pruebas que demuestran que la secuencia gramatical formada por el nombre y su complemento da lugar a un constituyente de naturaleza distinta a aquel otro constituyente en el que aparecen los adjuntos ${ }^{8}$. Estas pruebas serán las siguientes: la sustitución por una forma pronominal, el orden de palabras y la coordinación.

Una de las pruebas empíricas que con más eficacia ayuda a descubrir la estructura que subyace a una expresión lingüística es la sustitución. Un factor que hay que tener en cuenta es que esta transformación está condicionada precisamente por exigencias de tipo estructural. Así, se mantiene que únicamente las unidades que se organizan como constituyentes pueden ser sustituidas por un elemento. Además, otro requisito impuesto por

\footnotetext{
${ }^{7}$ Esquema formal tomado de Radford (1988:229).

${ }^{8}$ Nótese que en el formato de (13) no se precisa la posición de adjunto. En este tipo de representación, en el que se pretende hacer constar únicamente los distintos niveles de proyección que se observan en una categoría sintagmática, esta información es irrelevante. En efecto, la unidad en la que aparece el adjunto, sea cual sea su nivel (véase Radford (1988:255-8)), se proyecta en otra unidad del mismo orden jerárquico. Como base para nuestra exposición optaremos por situar este elemento en la categoría intermedia, disposición que puede considerarse como la más normal:
} 
dicha transformación es la necesidad de que los elementos implicados en la misma sean de igual naturaleza. Al decir de Halliday y Hasan (1976:89-90):

(14) ... as a general rule, the substitute item has the same structural function as that for which it substitutes.

Entendidas de este modo las cosas, es fácil deducir la pertinencia de esta operación en el tema que nos ocupa. Puesto que, como hemos advertido, sirve para identificar los componentes de una secuencia gramatical que contraen entre sí relaciones sintagmáticas de una misma clase, garantiza la posibilidad de distinguir dos posiciones estructurales diferentes como son las de complemento y adjunto. Busquemos ahora la ratificación de las ideas expuestas por medio de datos fundados en la observación de la realidad lingüística.

Vamos a tratar de determinar, en primer lugar, el elemento que puede reemplazar la proyección nominal intermedia, categoría en la que se incluyen tanto los complementos como los adjuntos del núcleo. Acogiéndonos a la propuesta más corriente en los trabajos elaborados desde el marco de la Teoría de la X-barra, consideraremos que esta categoría es sustituible por la proforma one $e^{9}$. A continuación ofrecemos tres oraciones que contienen unas secuencias nominales, las que hemos destacado en cursiva, que difieren en lo que toca a la aceptación de la sustitución por esta proforma:

(15) (a) The adoption of an austere economic policy in a period of crisis in Germany is not as troublesome as the one in England.

(b) The adoption of an austere economic policy in a period of crisis is not as troublesome as the one in a period of prosperity.

(c) *The adoption of an austere economic policy is not as troublesome as the one of a belligerent foreign policy.

${ }^{9}$ Tampoco faltan alternativas en esta área. Herbst (1988:275-7), por ejemplo, recurrió a las formas someone y something. El problema que presenta su sugerencia, sin embargo, es que estos elementos pueden aparecer también en lugar de la proyección nominal máxima. La no constatación de este hecho puede llevar a describir erróneamente la esencia de algunas estructuras. Es lo que creemos que ocurre en el siguiente ejemplo:

I heard a concerto by Vivaldi on the radio.

donde, tras la aplicación de esta prueba, Herbst (1988:276) postula el carácter adjunto del grupo preposicional on the radio:

I heard something on the radio - a concerto by Vivaldi.

*I heard something by Vivaldi - a concerto on the radio.

cuando, en realidad, este grupo preposicional y la secuencia nominal que le precede son estructuralmente independientes. Por otra parte, ciñéndonos a cuestiones relacionadas con el sustituto que nosotros hemos elegido, es necesario advertir que éste se ha de distinguir de otras dos proformas one homomorfas: una sería el numeral y otra sería aquella que sirve como sustituto de todo un grupo nominal. Las peculiaridades formales y funcionales de cada una de ellas son perfectamente explicadas por Quirk et alii (1985:386-8 y 869-70). 
Observamos que mientras que en (15.a) y (15.b) la sustitución está permitida, en (15.c) este procedimiento origina una construcción agramatical. Como es de esperar, el distinto estatus jerárquico de los elementos reemplazados será la causa fundamental de tales desemejanzas. En efecto, el que la sustitución se haya podido llevar a cabo en las dos primeras oraciones es un claro indicio de que las unidades ahí examinadas constituyen una proyección intermedia. En la última oración, en cambio, la operación no es viable por cuanto no se ha seleccionado una categoría apropiada para su aplicación. Veamos las consecuencias que se derivan de los datos que poseemos en lo concerniente a la delimitación de la identidad de los complementos y los adjuntos de un nombre.

El análisis que hemos hecho del fenómeno de la sustitución ha revelado, en primer lugar, que el complemento y el adjunto forman parte de unidades sintácticas de la misma naturaleza categorial, $N^{\prime}$. De todos modos, se ha podido acreditar que hay una diferencia evidente entre ambos. Ésta atañe a la esencia del elemento con el que se agrupan. Los adjuntos, por un lado, se combinan con una proyección nominal intermedia, lo cual explica que en (15.b) la exclusión del grupo preposicional in a period of crisis de la estructura sustituida no afecte a la buena construcción de la oración. Los complementos, sin embargo, se unen a la categoría de nivel cero, lo que impide la producción de construcciones como la de (15.c). A la vista de estos hechos, podemos concluir que será no el que un elemento sea reemplazado por one sino el que no pueda coaparecer con esta proforma lo que le otorgará el rango de complemento. Esto es lo más importante, dado que refleja el hecho de que los complementos son los constituyentes más próximos al núcleo de un sintagma nominal.

Por último, debemos hacer hincapié en las palabras de algunos lingüistas acerca de las lagunas que tiene esta prueba. Ello nos ayudará a calibrar mucho mejor el valor de la misma. Las críticas a las que nos vamos a referir se han dirigido principalmente en dos direcciones. Así, hay autores que cuestionan la validez de este procedimiento como mecanismo que permite aislar estos constituyentes sintácticos. Otros, en una actitud no tan desfavorable hacia dicho procedimiento, hacen notar solamente que el mismo no tiene autoridad suficiente para definir todo el conjunto de unidades que se consideran complementos.

Entre los lingüistas que se adhieren a la primera propuesta se encuentra Napoli (1993:424-5). Esta autora indica que la posibilidad de sustitución de un elemento por una proforma no constituye un argumento acertado para apoyar la idea de la existencia de distintos niveles de proyección en la estructura de una unidad sintagmática. El inconveniente que, según ella, presenta este criterio es que las proformas, al ser elementos de naturaleza semántica, pueden corresponderse con unidades que no forman un bloque sintáctico. Aunque no tenemos intención de entrar a analizar esta afirmación en toda su extensión, sí nos interesa examinar su adecuación en el terreno que nos ocupa que, además, es en el que se mueve esta investigadora en las páginas en las que está tratando este tema. 
Su hipótesis es que one es una de las proformas con las que puede producirse el hecho anteriormente descrito. Para justificar lo que dice, Napoli (1993:425) aporta el siguiente ejemplo:

(16) John bought a new car and it has those mag wheels I've been dying to get. So I went out and finally bought one, too.

donde, como ella misma explicita, one nos remite a la secuencia a new car with mag wheels, que no se ha comportado como un constituyente sintáctico en el enunciado precedente. Ella piensa que esto es lo que desvirtúa el criterio que estamos considerando. Sin embargo, en nuestra opinión, esta autora está equivocada en su razonamiento. El problema que presenta su planteamiento es que la proforma one que nosotros hemos utilizado en nuestra argumentación y la que ella emplea en la suya tienen un estatus muy diferente, como lo demuestra, entre otras cosas, el hecho de que esta última sea utilizada en lugar de todo un sintagma nomina ${ }^{10}$. Este detalle, que esta autora no ha parecido advertir, determina que sus predicciones sean incorrectas. Esta circunstancia nos obliga a rechazar su propuesta.

Más moderada que la de esta autora es la posición de Jackendoff (1977:59 y 61, nota 4). Este autor, que acepta el criterio, hace notar que no todas las secuencias con una estructura nombre + complemento son distinguidas por el mismo. Para él, los casos más claros en los que los resultados obtenidos con este recurso son correctos son aquellos en los que los complementos se realizan mediante sintagmas preposicionales introducidos por la preposición of. Pero, por otro lado, señala también que hay una serie de complementos que pueden coexistir con la proforma one. Por tanto, cualquier estudio que se base en la aceptación de este criterio en términos absolutos presentaría una visión muy restringida del fenómeno de la complementación nominal. Con el fin de aclarar este punto ofrece los ejemplos de (17):

(17) (a) Arguments with Bill are less fruitful than ones with Harry.

(b) Arguments with many premises are less impressive than ones with few premises.

donde tanto el complemento en (17.a), with Harry, como el adjunto en (17.b), with few premises, comparten la capacidad de coaparecer con la proforma. Todo lo cual viene a significar, como este autor explica, que esta prueba nos proporciona una condición si bien no necesaria sí, al menos, suficiente para la identificación de los complementos de una forma nominal.

Otro artificio al que se recurre para evidenciar las diferencias estructurales entre los complementos y los adjuntos es el estudio del ordenamiento lineal de las unidades que

\footnotetext{
${ }^{10}$ Véanse a este respecto los comentarios que realizamos en la nota 9.
} 
conforman una expresión lingüística. Este recurso establece que cuando en dicha expresión el núcleo se halla modificado por un complemento y un adjunto, el núcleo y el complemento han de aparecer contiguos. Como vamos a tener oportunidad de observar inmediatamente, este hecho se puede poner en relación con el análisis que hace la Teoría de la X-barra de la constitución de una categoría sintagmática.

Veamos ahora algunos de los ejemplos que han propuesto los lingüistas que se muestran partidarios de este criterio:
(18) (a) a student of physics with red hair
(b) *a student with red hair of physics ${ }^{11}$
(19) (a) a king of England of considerable intelligence
(b) *a king of considerable intelligence of England ${ }^{12}$
(20) (a) John described his discomfort with the direction of the investigation from the beginning.
(b) *John described his discomfort from the beginning with the direction of the investigation ${ }^{13}$.

En el primer ejemplo de cada par el complemento, en negrita, antecede al adjunto, en cursiva. En el segundo ejemplo de cada par, sin embargo, estos dos elementos han intercambiado sus posiciones.

La confirmación de que la disposición en la que se encuentran los elementos de las secuencias (a) es la correcta es, como hemos anunciado en el párrafo con el que iniciábamos nuestro examen de este criterio, otro argumento en favor del esquema de proyección recogido en (13). En éste, recordemos, el núcleo y el complemento se colocaban en el estrato más bajo dentro de lo que era la organización jerárquica de un constituyente sintagmático. El hecho de que secuencias como las de (b) no se puedan elaborar queda asegurado por una restricción con la que se excluyen marcadores sintagmáticos en los que las líneas que los representan se cruzan. Radford (1988:121) formula este requisito estructural del siguiente modo:

(21) If one node $\mathrm{X}$ precedes another node $\mathrm{Y}$, then $\mathrm{X}$ and all descendants of $\mathrm{X}$ must precede $\mathrm{Y}$ and all descendants of $\mathrm{Y}$ ( $\mathrm{A}$ is a descendant of $\mathrm{B}$ iff $\mathrm{A}$ is dominated by B)

Así, por ejemplo, como en (18.b) esta condición no se ha cumplido, obtenemos una expresión que no está bien formada:

\footnotetext{
${ }^{11}$ Ejemplos tomados de Freidin (1992:42).

${ }^{12}$ Ejemplos tomados de Huddleston (1984:264).

${ }^{13}$ Ejemplos tomados de Hendrick (1981:887).
} 
(22)

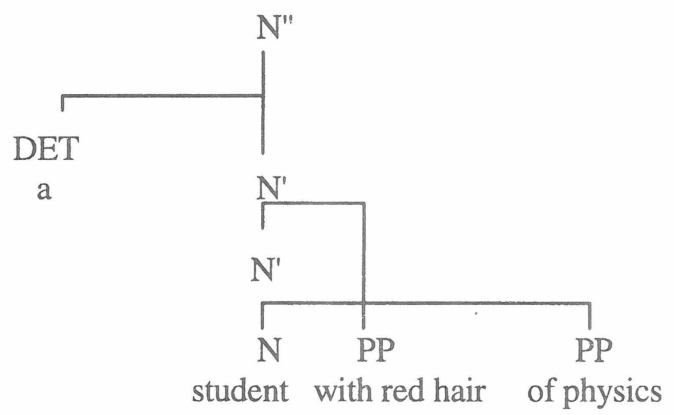

A pesar de lo señalado hasta este momento, este criterio necesita unas apostillas que, de no ser tenidas en cuenta, nos pueden llevar a una deficiente aplicación del mismo. Las dificultades que se nos pueden presentar al llevarlo a la práctica radican en la existencia de otros factores, distintos al que nosotros consideramos, que también regulan la colocación de los elementos de una expresión lingüística. Seguidamente nos ocuparemos de algunos de ellos y explicaremos cómo pueden influir en la cuestión que estamos tratando.

El primero de los factores sobre el que vamos a llamar la atención es aquel que fija el orden en función de exigencias comunicativas. Estas exigencias pueden venir marcadas por la prominencia que se le quiera dar a uno de los elementos de los que consta la expresión o por su complejidad. Estos dos aspectos se hacen evidentes en la estructuración de todas las unidades sintácticas, ya sean de naturaleza clausular o sintagmática. Ahora bien, si comparamos sus efectos en las secuencias nominales con los que producen en las cláusulas advertiremos que éstos son más acusados en aquéllas, donde, como Quirk et alii (1985:1398) manifiestan, los patrones que definen la disposición de sus constituyentes son menos rígidos. Pasemos a describir con ejemplos cada una de estas situaciones.

Un procedimiento mediante el que se consigue dar relevancia a un elemento es el de situarlo en la posición final de la estructura sintáctica de la que forma parte. Así, en los siguientes ejemplos de Quirk et alii (1985:1398) se puede observar que todos los integrantes de una secuencia nominal pueden ser realzados con ayuda de este mecanismo:

(23) (a) Lovell's discovery of the new star in 1960

(b) The discovery by Lovell in 1960 of the new star

(c) The discovery of the new star in 1960 by Lovell

(d) (?) Lovell's 1960 new star discovery

La otra situación en la que nos vamos a detener es la que concierne a unidades cuya estructura es demasiado intrincada. Con relación a la misma hemos de decir que uno de los principios por el que se rige el acto comunicativo establece que éstas sean ubicadas al final para facilitar la comprensión del mensaje. Además de los citados Quirk et alii (1985:1398-9), otra autora que considera este aspecto es Anderson (1979:64, nota 1), quien sobre la base de pares de ejemplos como el que sigue: 

(a) Mary's analysis on Wednesday of the samples
(b) Mary's analysis on Wednesday of the 13 contaminated examples

comenta que:

(25) ... the examples are better when the direct object NPs are longer, suggesting that there has been a stylistic movement of a complex NP.

Hasta aquí nuestra reflexión acerca de la acción de este factor informativo que organiza la composición de los sintagmas nominales. Únicamente nos resta indicar cómo puede afectar su actividad a la aceptación del criterio que estamos examinando. En este sentido, tenemos que señalar que su efecto es positivo por un doble motivo. Primero, porque sirve para afianzar la idea de que los elementos de este tipo de sintagmas no se disponen arbitrariamente ${ }^{14}$. Segundo, porque se puede considerar que las estructuras que resultan de la acción de este factor son marcadas, quedando garantizada la utilidad del criterio en las estructuras no marcadas o básicas.

Un factor que sí nos puede plantear serios problemas a la hora de hacer uso de este criterio es el hecho de que en algunas ocasiones el ordenamiento de los elementos calificados de adjuntos está determinado también por unas reglas. El resultado es que podría pensarse en el carácter completivo del adjunto más próximo al núcleo nominal. Herbst (1988:275, nota 15) alude certeramente a esta circunstancia empleando estas secuencias como ejemplo:
(26)
(a) a lecture at 4 p.m. on Tuesdays
(b) ?a lecture on Tuesdays at 4 p.m.

La dudosa gramaticalidad de (26.b) justificaría un análisis del grupo preposicional at 4 p.m. como complemento, lo cual sería equivocado. Consiguientemente, desde esta perspectiva habría que decir que esta prueba no es muy operativa. Su utilidad, después de todo lo que hemos expuesto, se ve solamente en aquellos casos en los que coaparecen un complemento y un adjunto. Pero, esta condición supone que éstos tienen que ser previamente identificados por medio de otro procedimiento.

El último mecanismo al que nos vamos a referir para distinguir los complementos de los adjuntos es la coordinación. Antes de verificar su efectividad hemos de señalar que si bien esta prueba permite constatar, al igual que las anteriores, que los complementos y adjuntos nominales manifiestan propiedades estructurales dispares, en cambio no apunta nada acerca de la esencia de las mismas.

\footnotetext{
${ }^{14}$ Opinión que es defendida, por ejemplo, por Napoli (1993:135).
} 
Esta prueba es usada por Radford (1988:190), quien para justificar su validez se apoya en un requisito que deben cumplir las unidades de la lengua a las que afecta la operación de coordinación: su compatibilidad sintáctica. Los ejemplos con los que defiende su postura son éstos:
(a) a student [of Physics] and [of Chemistry]
(b) a student [with long hair] and [with short arms]
(c) *a student [of Physics] and [with long hair]
(d) *a student [with long hair] and [of Physics]

Estos datos ponen de relieve que sólo en (27.a) y (27.b) los elementos que se coordinan, de índole completiva, en un caso, y adjunta, en el otro, poseen características estructurales idénticas.

La efectividad de esta prueba tiene que ser enjuiciada con arreglo a estos dos parámetros: por una parte, su utilidad para descubrir que los complementos y los adjuntos son elementos estructuralmente diferentes; y, por otra parte, su autoridad para delimitar el campo de los adyacentes nominales que muestran uno u otro comportamiento funcional. En este sentido, la situación que se nos presenta es muy parecida a la que teníamos planteada en la prueba anterior. Así, como hemos podido comprobar, la prueba es adecuada para lograr el primero de los objetivos. Sin embargo, los problemas surgen cuando se la considera con relación al segundo tema. Veamos por qué.

Para dar respuesta a esta pregunta es interesante estudiar los factores de los que depende la coordinación. Un factor importante, según lo expuesto, es el de la compatibilidad sintáctica entre las unidades que se unen por medio de este mecanismo. Pero, por otro lado, intervienen igualmente factores semánticos e incluso pragmáticos ${ }^{15}$. Pues bien, este hecho es clave para fundamentar nuestra negativa a admitir esta prueba como un criterio que contribuya satisfactoriamente a la categorización funcional de las unidades lingüísticas. Así, sucede que muchas veces, por causas que obedecen a alguno de los otros factores que acabamos de señalar, unidades que tienen el mismo estatus sintáctico no se pueden coordinar. Obsérvese que la coordinación de adjuntos en los ejemplos de (28) produce construcciones inaceptables:
(28)
(a) *the student for the job and from Scotland
(b) *the delegate with big hands and at the conference

\footnotetext{
13 Una alusión al papel desempeñado por estos otros condicionantes la hallamos en los trabajos de Mackenzie (1983:36-7), Quirk et alii (1985:941-73) y Fernández Lagunilla y Anula Rebollo (1995:310-3). También Bosque Muñoz (1989:45-6) nota las deficiencias de los estudios sobre la coordinación en los que únicamente se contempla el factor funcional.
} 
(c) $*$ the bus that fell into the river and hit the traffic lights ${ }^{16}$

Por tanto, como ocurriera con la prueba del ordenamiento de palabras, llegamos a la conclusión de que este procedimiento no distingue específicamente entre complementos y adjuntos. Por este motivo, opinamos que es necesario guiarse por otros criterios en esta cuestión.

Resumiendo, pues, podemos decir que la totalidad de los datos que han sido examinados en esta sección demuestran que existen diferencias cualitativas entre los complementos y los adjuntos. Según se ha podido ver, el esquema de proyección categorial que se propugna en la Teoría de la X-barra refleja puntualmente esta circunstancia. Otra cosa muy distinta es que las pruebas que hemos revisado puedan ser tomadas como criterios útiles para describir el valor funcional de una determinada secuencia lingüística. Éste es un tema respecto al cual hemos sido bastante escépticos. Así, hemos visto, por un lado, que el criterio de la pronominalización da pie a una visión muy limitada del fenómeno de la complementación nominal. Por otra parte, el criterio del orden de palabras y el de la coordinación pueden acarrear descripciones completamente erróneas.

No queremos concluir esta parte de nuestro trabajo dedicada a examinar las desemejanzas estructurales entre los complementos y los adjuntos sin antes dar cuenta de otra razón que se ha aducido para confirmar la existencia de tales desemejanzas. Ésta viene a mostrar que esta distinción del nivel sintáctico tiene un correlato semántico. Hornstein y Lightfoot (1981:21) entienden esta correlación de la siguiente forma ${ }^{17}$ :

(29) We also assume that syntactic constituent structure will play a role in determining the semantics of Noun Phrases, and specifically that each N-bar specifies a 'semantic property'.

Estos autores ilustran el hecho enunciado con los ejemplos recogidos en (30):

(30) (a) John is a $\left[\mathrm{N} \cdot \mathrm{N}_{\mathrm{N}}\right.$ student $]$ of Physics $]$.

(b) John is a $\left.\left.\mathrm{N}_{\mathrm{N}} \cdot \mathrm{N}_{\mathrm{N}} \cdot \mathrm{N}_{\mathrm{N}} \mathrm{student}\right]\right]$ with long hair].

Como vamos a ver, el modo tan distinto en el que cada uno se ha segmentado manifiesta la disparidad en las relaciones semánticas que se establecen entre sus integrantes ${ }^{18}$.

\footnotetext{
${ }^{16}$ Nótese que la agramaticalidad de esta secuencia nominal se pone de manifiesto sólo cuando las dos acciones con las que se restringe la denotación del nombre bus describen distintos momentos de un mismo suceso. En este caso el orden en el que se desarrollan los acontecimientos condiciona la distribución de los componentes coordinados. En cambio, si los dependientes del nombre hubiesen señalado hechos entre los qụe no hay ninguna conexión, la construcción en cuestión habría sido correcta.

${ }^{17}$ Esta cita la hemos tomado de Radford (1988:188).

${ }^{18}$ Sobre este tema véase, por ejemplo, Jackendoff (1977:57).
} 
Consideremos primero (30.a). El complemento está directamente emparentado con el núcleo del sintagma. Este mismo tipo de intimidad se produce entre los homólogos de estas unidades en el plano semántico. Recuérdese que los argumentos aparecen en la caracterización de la noción léxica expresada por el predicado. Consiguientemente, puede decirse que el dato aportado por of Physics forma parte de la unidad informativa constituida en torno a student con la que se individualiza la persona de John.

Examinemos en segundo lugar (30.b). En este caso el adyacente nominal, al funcionar como adjunto, se vincula a la categoría sintagmática intermedia. Igualmente, el análisis semántico de esta secuencia muestra divergencias con el de la anterior. La información aportada por with long hair constituye una especificación de la entidad de la que hablamos que se ha de unir a la realizada previamente por student. Son dos, por tanto, los rasgos con los que se delimita la identidad de John.

La prueba del paradigma puede ser utilizada para corroborar las afirmaciones que estamos realizando. Así, obsérvese que sólo en este último ejemplo el nombre student se halla en paradigma contrastivo con nombres absolutos:

(31) (a) *John is a [N $\left[{ }_{N} m a n\right]$ of Physics].

(b) John is a $\left.\left.{ }_{\mathrm{N}} \cdot \mathrm{N}_{\mathrm{N}} \cdot \mathrm{N}_{\mathrm{N}} \mathrm{man}\right]\right]$ with long hair].

De la misma manera, este desigual carácter de los modificadores nominales se manifiesta de manera palpable al describir secuencias con múltiples interpretaciones en lo que toca a su contenido y a su estructura sintáctica. Como ejemplificación recogemos la construcción a la que Radford (1988:188) recurre para explicar esta situación:

(32) a student of high moral principles

Este autor observa que el doble sentido que tiene la citada construcción:

(33) (a) a person who studies high moral principles

(b) a student who has high moral principles

se corresponde con una doble posibilidad de análisis en el plano sintáctico:

(34) (a) a [N $[\mathrm{N}$ student] of high moral principles]

(b) a $\left[_{N}\left[{ }_{N}\left[{ }_{N} s t u d e n t\right]\right]\right.$ of high moral principles $]$

Lo importante es, pues, advertir que en un grupo nominal las diferencias en el tipo de relación sintáctica que un nombre establece con sus dependientes determinan muy significativamente la manera en la que dicho grupo nominal va a ser interpretado. 
Por último, otro de los criterios en el que se basa esta distinción entre complementos y adjuntos es el que atiende a las restricciones que una unidad léxica cualquiera impone sobre sus dependientes en lo que respecta a la capacidad que éstos tienen para aparecer en un contexto determinado. En concreto, este criterio estipula que mientras que los adjuntos se pueden acumular en una construcción sintáctica sin cortapisas, los complementos no. Esta propiedad de los adjuntos y de los complementos no hace sino poner de manifiesto la autonomía de la que disfrutan los primeros con relación al núcleo al que están subordinados. Por el contrario, la elección de los complementos se halla sujeta a las exigencias léxicas del núcleo de la secuencia en la que aparecen.

La revisión de algunos de los trabajos que se han realizado sobre la complementación desde el marco de la Gramática de Dependencias, bien sea en términos generales o centrados en una categoría gramatical específica, nos ha permitido ver que se trata de un criterio bastante usado. Así, autores como Engelen (1975) ${ }^{19}$, Matthews (1981:127 y 152-3), Allerton (1982:39), Herbst (1988:269) y Comesaña Rincón (1989:80) lo han tenido en cuenta en sus descripciones. Del mismo modo, en estudios que se efectúan según los postulados de otros modelos lingüísticos se refleja este mismo hecho. Es el caso, por ejemplo, de las propuestas de Günther (1978:134-5), emitida desde el marco de la Gramática Categorial, y de Zubizarreta (1987:53) y Graña López (1994:106), ambas formuladas en el ámbito de la Gramática Generativa ${ }^{20}$.

Examinemos, pues, la pertinencia de esta prueba en la determinación del estatus funcional de los adyacentes nominales. Tal como hemos hecho en las secciones anteriores, empezaremos por exponer, en sus líneas más generales, la propuesta de estos autores, para después entrar a comentar los aspectos más controvertibles de la misma.

Consideremos las siguientes secuencias nominales en las que los segmentos que desempeñan el papel de complemento aparecen en negrita y los que funcionan como adjunto lo hacen en cursiva:

(35) (a) The performance of a well-knnown play in Madrid at seven o'clock

(b) *The show of a well-known play

(c) The show in Madrid at seven o'clock

\footnotetext{
${ }^{19}$ Este autor se refiere a este recurso con el nombre de prueba de especifidad. De acuerdo con este enunciado, considera los complementos constituyentes específicos mientras que analiza los adjuntos como constituyentes no específicos.

${ }^{20}$ Para estos últimos investigadores el principio que regula el fenómeno que tratamos es la Condición de los Papeles Temáticos. Así es como Chomsky (1981:36) lo definió: "Each argument bears one and only one $\theta$-role, and each $\theta$ role is assigned to one and only one argument". Es, concretamente, la segunda cláusula de este precepto la que controla el modo en el que se ha de obrar en este terreno.
} 
(36) (a) *The performance of a well-known play of a piece of music in Madrid at seven o'clock

(b) The performance of a well-known play in a theatre in Madrid at seven o'clock next Saturday

La explicación de los motivos sobre los que se basan nuestras apreciaciones acerca de la gramaticalidad o agramaticalidad de todas estas expresiones lingüísticas nos permitirá apreciar las propiedades de los complementos y adjuntos que nos interesa resaltar.

Veamos primero los ejemplos de la serie (35). Encontramos dos nombres, performance y show, que designan un espectáculo de entretenimiento. Sin embargo, además de este aspecto de su denotación que estos nombres comparten, hay otro factor que los distingue. Así, mientras que ni en (35.a) ni en (35.c) existen restricciones en relación con el empleo de dependientes que señalen el lugar y el momento en el que tiene lugar dicho espectáculo, sólo en (35.a) es posible la presencia de un adyacente que represente la entidad en torno a la cual gira el mismo. Es evidente, pues, que sintácticamente el vínculo que se establece entre un nombre y su complemento es más estrecho que el que se establece entre un nombre y su adjunto. Como la situación que aquí se ha mostrado nos ha permitido observar, la capacidad de aparecer con un complemento es un rasgo peculiar de cada nombre. Los adjuntos, por el contrario, se caracterizan por poder insertarse libremente en cualquier construción.

En cuanto a los ejemplos de (36), podemos comprobar otro rasgo diferencial que separa los complementos de los adjuntos. En consonancia con la idea central desarrollada en este apartado, observamos en (36.a) que es imposible también la coexistencia en una misma expresión nominal de dos complementos que indiquen el mismo tipo de argumento. Esto no ocurre con los adjuntos en (36.b). En este ejemplo el nombre es modificado por cuatro adyacentes que se agrupan en dos campos conforme al aspecto de la denotación del nombre que especifican, en este caso el lugar y el tiempo donde se celebra el espectáculo. Ello se debe a que, a diferencia del adjunto, el complemento expresa toda la información relativa a la unidad léxica de la que depende a la que se puede hacer referencia.

Todo lo expuesto hasta aquí nos hace pensar que el criterio que estamos considerando resuelve con acierto el problema de la delimitación entre las unidades que estudiamos. Sin embargo, hemos de dejar constancia de que no todos los lingüistas comparten esta opinión. En efecto, pese a lo comentado, hay una serie de autores (Vater (1978:25-8), Somers (1984:515) y Herbst (1984:2-3), entre otros) que entienden que este criterio no identifica de manera inequívoca los complementos y los adjuntos de una pieza léxica. A continuación presentamos algunos de los argumentos que se ofrecen más frecuentemente en contra del mismo.

Uno de ellos es que las posibilidades de aparición de los adjuntos en los grupos nominales también se encuentran en cierta medida restringidas. Por ejemplo, la no aceptabilidad de: 
ha sido motivada por la presencia del sintagma preposicional with an axe, que denota el instrumento con el que se realiza el proceso expresado por toda la secuencia nominal. En este caso, según esta prueba, este constituyente debería ser catalogado como complemento. Sin embargo, como veremos unas líneas más adelante, lo correcto es analizarlo como adjunto.

Otro argumento que se aduce para cuestionar este criterio afecta, en particular, a la segunda de las situaciones que expusimos antes. Decíamos, recuérdese, que era imposible el emplazamiento en una expresión lingüística de dos o más complementos que saturasen la misma variable argumental. Añadíamos que, por el contrario, era factible la acumulación en dicha expresión lingüística de aỏjuntos que modificasen el mismo aspecto (temporal, espacial, etc.) de la denotación de su núcleo. Sin embargo, ejemplos como los que aportamos ahora demuestran que la gramática también regula el empleo repetido de los adjuntos en estas circunstancias que nos ocupan:

(a) *Pete destroyed the city on Tuesday on Wednesday.

(b) *Pete destroyed the city with Joe with $\mathrm{Sue}^{22}$.

Utilizando este procedimiento, consiguientemente, realizaríamos una descripción errónea de las secuencias on Tuesday y with Joe.

No hay ninguna duda de que la tesis de estos autores tiene su valor, pero nos parece que una distinción entre complementos y adjuntos apoyada en este criterio sigue teniendo sentido. Naturalmente, esto debe pasar por una concepción del mismo que permita mejores resultados. Para ello, resultará conveniente analizar los factores que regulan la distribución de ambas clases de elementos.

Previamente hemos establecido que la propiedad que tiene un determinado lexema nominal de aparecer con complementos es una característica individual de dicho lexema nominal. Quiere esto decir que tanto el número, como el tipo, como los rasgos semánticos de los complementos vienen marcados independientemente por la pieza léxica a la que modifican. Desde este punto de vista, podemos apuntar que en la explicación de los contextos en los que aparece esta clase de adyacentes nominales hay una parte que pertenece a la morfosintaxis y otra que pertenece a la semántica.

${ }^{21}$ Ejemplo tomado de Mackenzie (1983:37).

${ }^{22}$ Ejemplos tomados de Grimshaw (1990:148). Ante tal evidencia, esta autora (1990:149) sugiere que el requisito comentado en la nota 20 de nuestro trabajo sea aplicado a todo el conjunto de los adyacentes nominales. Este acercamiento entre la sintaxis de los complementos y la de los adjuntos ha sido también asumido por autores como González Escribano (1991:238 y ss) y Graña López (1994:107). 
En cuanto a los adjuntos, las limitaciones que éstos pueden experimentar con respecto a una determinada forma nominal se presentan siempre de modo conjunto con otras formas nominales. En general, pues, el grado de influencia que ejerce un nombre sobre los adjuntos que tiene en su entorno es inferior al que ejerce sobre los complementos. Ello es consecuencia de que el uso de este tipo de unidades está condicionado por la naturaleza de la entidad que denota el nombre del que dependen. Así se puede justificar que, por ejemplo, no sean posibles secuencias como las de (37). En este caso, el nombre collapse, que expresa un proceso, no puede ir acompañado de un instrumental, ya que este tipo de elementos sólo es compatible con nombres que indican una acción o una posición ${ }^{23}$. Por supuesto que no siempre la exclusión de un adjunto está causada por las imposiciones que establece el nombre. Así, el que en (38) las dos construcciones sean agramaticales se debe a una anomalía en el plano semántico provocada por la combinación de dos grupos preposicionales que aportan información contradictoria. Por tanto, vistas las cosas de esta manera, podemos concluir que la distribución del adjunto tiene una base puramente semántica.

Con los datos que ahora poseemos creemos factible proponer una solución satisfactoria al problema que teníamos creado con relación a la validez del criterio de la libre adición de dependientes en la distinción complemento-adjunto. Hemos visto que existen restricciones en la aparición de una y otra clase de dependientes. Lo verdaderamente relevante en este punto, sin embargo, es que los tipos de restricciones observados en cada caso son distintos. Por este motivo, ha de redefinirse este criterio de tal modo que se puedan prevenir las ocasiones comentadas en las que la libre adición de adjuntos daba lugar a estructuras anómalas. En este sentido, nos adherimos a la propuesta de Matthews (1981:127), quien apunta que la posibilidad de ocurrencia de los adjuntos se halla "... free of any restriction but the need to make sense".

Aún habría que añadir otro obstáculo con el que tropieza el criterio que estamos considerando y que dificulta su aplicación. Ciertamente, podemos avanzar que el problema que en este momento vamos a plantear sí supone un auténtico inconveniente para aquellos que pretenden fundamentar su análisis de este aspecto gramatical en esta prueba exclusivamente. Veamos dónde se presenta la complicación a la que nos referimos.

Se ha señalado que uno de los rasgos que identifica los adjuntos es la facultad que tienen de ser incluidos en cualquier estructura sintáctica. El argumento que podemos oponer a esta indicación es que puede hacernos concebir la idea de que determinados tipos de elementos asumen siempre el rol de adjunto. Un ejemplo claro lo constituyen los adyacentes que determinan la situación ya sea local o temporal de una entidad cualquiera. En (35) y (36) evidenciamos que en los grupos nominales que nos remiten a un evento no existen restricciones respecto del uso de sintagmas preposicionales que nos ofrecen datos

\footnotetext{
${ }^{23}$ Las nociones proceso, acción y posición que acabamos de emplear deben ser entendidas según fueron definidas en el modelo de análisis funcional elaborado por Dik (1978:32 y ss). estudio.
} 
relativos al tiempo o lugar en el que tal evento ocurre. Esto no quiere decir que, a veces, con los nombres que implican determinaciones de esta naturaleza, estas unidades no deban ser consideradas complementos. Este hecho se manifiesta, por ejemplo, en secuencias como:

(39) (a) The President is on a trip to England.

(b) $\mathrm{He}$ is always talking about his pilgrimage to Shakespeare's birthplace.

ambas contienen un nombre, trip y pilgrimage, que requiere un elemento locativo que indique la dirección en la que se produce la expedición denotada por los dos nombres.

Por esta razón, esta prueba, aunque indicadora en un buen número de casos del estatus funcional de los adyacentes nominales, no garantiza siempre resultados satisfactorios. De ahí que tenga que verse acompañada de consideraciones de otra clase.

Como resumen de lo dicho en este trabajo, podemos destacar que en el mismo hemos intentado poner de relieve la complejidad que envuelve este asunto de la oposición entre las nociones de complemento y adjunto. En efecto, tras este examen que hemos llevado a cabo de algunos de los procedimientos que se han ideado para justificar tal dicotomía, queda claro que se echa de menos la existencia de instrumentos con capacidad suficiente para resolver adecuadamente esta cuestión en la totalidad de los casos que se someten a análisis. De hecho, de todos los que han sido explorados, solamente uno puede ser tenido en cuenta como criterio determinativo. Se trata del que repara en la índole latente de las unidades estudiadas. Por lo demás, las restantes pruebas revisadas presentan lagunas que hacen que tengamos dudas acerca de su validez.

Todo esto no significa, sin embargo, que la posibilidad de una clasificación de los adyacentes nominales realizada de acuerdo con los términos de esta dicotomía deba ser descartada. Acabamos de mencionar, por ejemplo, que hay un concepto, la latencia, en torno al cual se puede establecer esta distinción. Por otra parte, hemos comprobado que existe también una base estructural sobre la que fundamentar tal distinción. Finalmente, podemos notar que el vínculo que se establece entre las formas nominales y sus complementos, manifestado en las limitaciones que imponen los primeros a la aparición de los segundos, hace que la identificación de estos últimos resulte relevante para fijar subcategorías dentro de la categoría nominal. Por todo ello, pese a los innegables problemas que, como hemos apuntado, conlleva su aplicación, nos mostramos a favor de mantener la conocida dicotomía funcional. 
Tal decisión, además, la podríamos sustentar en la consideración de un último factor: el valor que estos elementos pueden tener como nociones teóricas ${ }^{24}$, esto es, como unidades que nos ayudan a describir determinados hechos lingüísticos. A este respecto, obsérvese la utilidad que estas dos nociones tienen en el desciframiento de las verdaderas relaciones estructurales que el nombre establece con la cláusula de infinitivo en las siguientes secuencias nominales isomórficas:
(40) (a) The man to vote
(b) The attempt to vote
(c) The proposal to vote

En (40.a) el modificador del nombre es un adjunto. La situación en (40.b) es completamente distinta. Aquí el modificador es un elemento de la complementación del nombre. Por último, en (40.c) nos hallamos ante un sintagma cuya ambigüedad estructural queda perfectamente explicada con señalar que el infinitivo puede ser catalogado bien como adjunto bien como complemento del nombre que lo precede.

\section{Referencias bibliográficas}

Allerton, D. J. (1982) Valency and the English Verb, Londres: Academic Press

Anderson, M. (1979) Noun Phrase Structure, Tesis Doctoral Inédita, Universidad de Connecticut

Bosque Muñoz, I. (1989) Las Categorías Gramaticales. Relaciones y Diferencias, Madrid: Síntesis

Chomsky, N. (1972) 'Remarks on nominalization' en Studies on Semantics in Generative Grammar, La Haya: Mouton Publishers, 11-61

Chomsky, N. (1981) Lectures on Government and Binding, Dordrecht: Foris

Chomsky, N. (1986a) Knowlegde of Language: Its Nature, Origin and Use, Nueva York: Praeger

Chomsky, N. (1986b) Barriers, Cambridge, Massachusetts: MIT Press

Colen, A. (1984) A Syntactic and Semantic Study of English Predicative Nominals, Bruselas: Koninklijke Academie

Comesaña Rincón, J (1989) 'Puntualizaciones teóricas al tratamiento de la complementación adjetiva en QUIRK `et alii' (1972) y QUIRK `et alii' (1985)', en Revista Española de Lingüística Aplicada 5, 73-98

Cook, V. J. (1988) Chomsky's Universal Grammar, Oxford: Basil Blackwell

\footnotetext{
${ }^{24}$ Con esta expresión traducimos el término "theoretical constructs" empleado por Radford (1988:55).
} 
Dik, S. C. (1978) Functional Grammar, Dordrecht: Foris Publications

Engelen, B. (1975) Untersuchungen zu Satzbauplan und Wortfeld in der geschriebenen deutschen Sprache der Gegenwart, Munich: Hueber

Fernández Lagunilla, M. y A. Anula Rebollo (1995) Sintaxis y Cognición: Introducción al Conocimiento, el Procesamiento y los Déficits Sintácticos, Madrid: Síntesis

Freidin, R. (1992) Foundations of Generative Syntax, Cambridge, Massachusetts: MIT Press

Garnica Silva, A. (1987) 'El verbo en la sintaxis X-bar' en El Verbo Inglés, Biblioteca de Estudios de Anglística n 13, Córdoba: Universidad de Córdoba, 167-92

Garrudo Carabias, F. (1991, 1996) Diccionario Sintáctico del Verbo Inglés, Barcelona: Ariel

González Escribano, J. L. (1991) Una Teoría de la Oración, Oviedo: Universidad de Oviedo, Servicio de Publicaciones

Graña López, B. (1994) Los Compuestos Verbales Ingleses, Tesis Doctoral Inédita, Universidad de Oviedo

Grimshaw, J. (1990) Argument Structure, Linguistic Inquiry Monograph N. 18, Cambridge, Massachusetts: MIT Press

Günther, H. (1978) 'Valence in categorial syntax', en W. Abraham (ed.) (1978) Valency, Semantic Case, and Grammatical Relations, Amsterdam: John Benjamins B.V., 12756

Halliday, M. A. K. y R. Hasan (1976) Cohesion in English, Londres: Longman

Hendrick, R. (1981) 'Subcategorization: its form and its functioning', Linguistics 19, 871910

Herbst, T. (1984) `Adjective complementation: a valency approach to making EFL dictionaries', Applied Linguistics 5/1, 1-11

Herbst, T. (1988) 'A valency model for nouns in English', Journal of Linguistics 24, 265 301

Hornstein, N. y D. Lightfoot (1981) Explanation in Linguistics, Londres: Longman

Horrocks, G. (1987) Generative Grammar, Londres: Longman

Huddleston, R. (1984) Introduction to the Grammar of English, Cambridge: C.U.P. Jackendoff, R. (1977) X' Syntax: a Study of Phrase Structure, Linguistic Inquiry Monograph N. 2, Cambridge, Massachusetts: MIT Press

Jespersen, O. (1909-49) A Modern English Grammar on Historical Principles, vol. II, Londres: Allen y Unwin

Leech, G. (1981) Semantics, Harmondsworth: Penguin

Lyons, J. (1968) Introduction to Theoretical Linguistics, Cambridge: C.U.P.

Lyons, J. (1977) Semantics, Cambridge: C.U.P. 
Mackenzie, J. L. (1983) 'Nominal predicates in a functional grammar of English', en S. C.

Dik (ed.) (1983) Advances in Functional Grammar, Dordrecht: Foris Publications, 31-51

Mackenzie, J. L. (1985) 'Nominalization and valency reduction', en A. M. Bolkestein, C. de Groot y J. L. Mackenzie (eds.) (1985) Predicates and Terms in Functional Grammar, Dordrecht: Foris Publications, 29-47

Matthews, P. H. (1981) Syntax, Cambridge: C.U.P.

Napoli, D. J. (1993) Syntax: Theory and Problems, Oxford: O.U.P.

Paul, P. (1982) 'Homonyms, semantic divergence and valency', Lingua 58, 291-307

Quirk, R. et alii (1985) A Comprehensive Grammar of the English Language, Londres:

Longman

Radford, A. (1988) Transformational Grammar, Cambridge: C.U.P.

Sells, P. (1985) Lectures on Contemporary Syntactic Theories: an Introduction to Government-Binding Theory, Generalized Phrase Structure Grammar, and LexicalFunctional Grammar, CSLI, Stanford University, Stanford: University of Chicago Press

Somers, H. L. (1984) 'On the validity of the complement-adjunct distinction in valency grammar', Linguistics 22, 507-30

Vater, H. (1978) 'On the possibility of distinguishing between complements and adjuncts', en W. Abraham (ed.) (1978) Valency, Semantic Case, and Grammatical Relations, Amsterdam: John Benjamins B.V., 21-45

Zubizarreta, M. L. (1987) Levels of Representation in the Lexicon and in the Syntax, Dordrecht: Foris 
